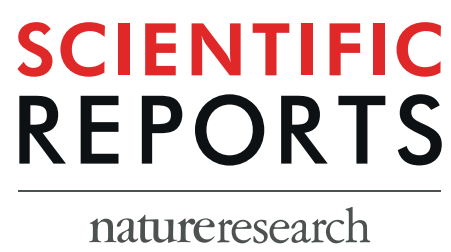

\title{
OPEN A change in the zinc ion concentration reflects the maturation of insulin-producing cells generated from adipose- derived mesenchymal stem cells
}

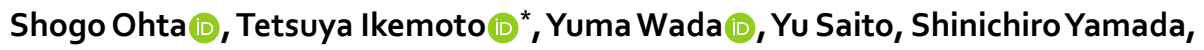 \\ Satoru Imura, Yuji Morine \& Mitsuo Shimada
}

The generation of insulin-producing cells (IPCs) from pluripotent stem cells could be a breakthrough treatment for type 1 diabetes. However, development of new techniques is needed to exclude immature cells for clinical application. Dithizone staining is used to evaluate IPCs by detecting zinc. We hypothesised that zinc ion $\left(\mathrm{Zn}^{2+}\right)$ dynamics reflect the IPC maturation status. Human adipose-derived stem cells were differentiated into IPCs by our two-step protocol using two-dimensional (2D) or 3D culture. The stimulation indexes of 2D -and 3D-cultured IPCs on day 21 were 1.21 and $3.64(P<0.05)$, respectively. The 3D-cultured IPCs were stained with dithizone during culture, and its intensity calculated by ImageJ reached the peak on day $17(P<0.05)$. Blood glucose levels of streptozotocininduced diabetic nude mice were normalised $(4 / 4,100 \%)$ after transplantation of 96 3D-cultured IPCs. $\mathrm{Zn}^{2+}$ concentration changes in the medium of $3 \mathrm{D}$ cultures had a negative value in the early period and a large positive value in the latter period. This study suggests that $\mathrm{Zn}^{2+}$ dynamics based on our observations and staining of zinc transporters have critical roles in the differentiation of IPCs, and that their measurement might be useful to evaluate IPC maturation as a non-destructive method.

The generation of insulin producing cells (IPCs) derived from pluripotent stem cells (PSCs) has the possibility to resolve the issue of donor shortages for islet transplantation in some countries ${ }^{1}$. Many studies have investigated the induction of IPCs from PSCs, such as embryonic stem cells, induced pluripotent stem cells, and other organ-derived cells ${ }^{2-11}$. In particular, adipose-derived stem cells (ADSCs) can be procured less invasively and without ethical problems compared with some types of PSCs ${ }^{12}$. An advantage of ADSCs is that up to 300-fold more stem cells can be obtained from the same quantity of bone marrow aspiration ${ }^{13}$. Moreover, they might be easy to apply clinically because such cell transplantation is considered auto-transplantation that does not require immunosuppression. Thus, we focused on ADSCs as a new cell source for IPCs ${ }^{12,14,15}$. We have previously reported a new two-step differentiation protocol ${ }^{16}$ and its modification to a more effective xeno-free and three-dimensional culture protocol ${ }^{17}$ to generate functional IPCs from ADSCs. For clinical application, it is important to exclude immature IPCs. Moreover, IPC maturation has been induced in vivo at a few months after transplantation ${ }^{3,18}$. Thus, the best timing for IPC transplantation is still unclear.

The role of the zinc ion $\left(\mathrm{Zn}^{2+}\right)$ in pancreatic $\beta$-cells is important for basic cell structures and enzymes. Mammalian pancreatic $\beta$-cells contain higher levels of intracellular $\mathrm{Zn}^{2+}$ than other organs ${ }^{19}$. In $\beta$-cells, insulin binds to $\mathrm{Zn}^{2+}$ and is converted into a hexamer within secretory granules ${ }^{20}$. Then, $\mathrm{Zn}^{2+}$ is released with insulin and absorbed by $\beta$-cells again. Therefore, mature $\beta$-cells both take up and $\mathrm{Zn}^{2+}$ secrete ${ }^{21,22}$. Dithizone staining is used to detect islets by the presence of high density $\mathrm{Zn}^{2+}$, which can be applied to evaluate IPCs ${ }^{8,16,20}$. However, cells are destroyed by the toxicity of dithizone in this procedure, and it is dangerous to use dithizone clinically because of its carcinogenicity ${ }^{8,20}$. 

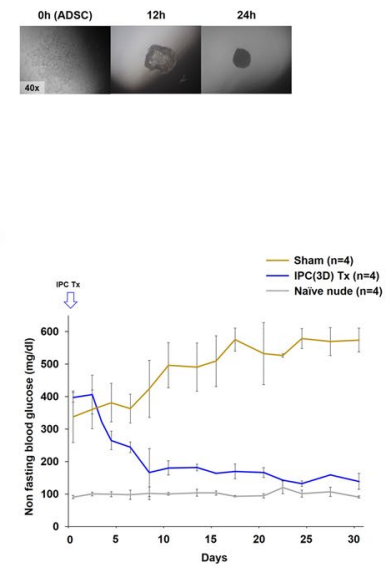
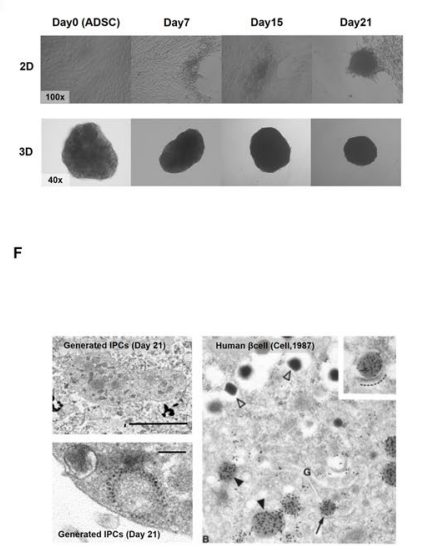
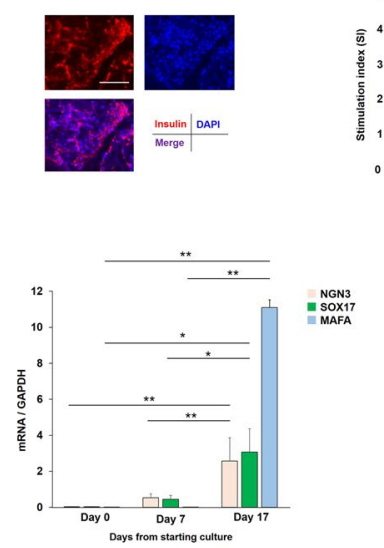

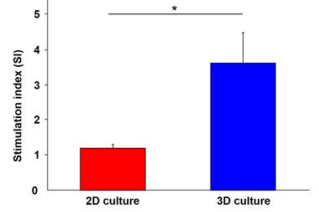

H

Figure 1. (A) ADSCs formed cell clusters within 24 hours after adding $\mathrm{RCP} \gamma$ pieces. Original magnification $\mathrm{x} 40$. (B) Cell cluster-like formation required more time in conventional 2D culture (upper row, original magnification $\mathrm{x} 100$ ) compared with 3D culture (lower row, original magnification $\mathrm{x} 40$ ). (C) Stimulation index of 3D-cultured IPCs was significantly higher than that of 2D-cultured IPCs on day 21 ( $* P=0.004$, Welch's test). (D) Mature IPCs expressed insulin strongly according to immunofluorescence staining. Day 21, 3D-cultured IPCs. (E) Blood glucose levels of streptozotocin-induced diabetic nude mice changed to normoglycaemia levels after IPC transplantation (blue line, $\mathrm{n}=4$ ), whereas the sham mouse group (ochre line, $\mathrm{n}=4$ ) did not reach a normal range. Grey line shows blood glucose levels of naïve nude mice $(n=4)$. (F) Electron microscopy revealed dense cystic microstructures (left, upper row. Scale bar, $5 \mu \mathrm{m}$ ) and secretory granule-like structures (left, lower row. Scale bar, $0.2 \mu \mathrm{m}$ ) in the cytoplasm of 3D-cultured IPCs on day 21, resembling secretory granules observed in human naïve $\beta$-cell ${ }^{25}$ (right). White arrowhead: mature granules; black arrowhead: clathrin-coated secretory vesicles; arrow: condensing secretory material in a clathrin-coated Golgi cisterna. (G) RT-PCR analyses of SOX17, NGN3, and MAFA expression on days 0 , 7. and $17(* P<0.01, * * P<0.001$, Bonferroni's test). (H) Immunohistochemistry of 3D-cultured IPCs on day 21 showed strong insulin expression (arrowhead). R, RCP $\sim$ piece. Scale bar, $25 \mu \mathrm{m}$.

\begin{tabular}{|l|l|l|l|l|}
\hline & Base line $(\mathbf{p M})$ & Low glucose $(\mathbf{p M})$ & High glucose $(\mathbf{p M})$ & SI \\
\hline Conventional culture $(2 \mathrm{D}, \mathrm{n}=3)$ & $18.1 \pm 2.0$ & $7.5 \pm 2.6$ & $8.9 \pm 2.7$ & $1.2 \pm 0.1$ \\
\hline 3D culture $(\mathrm{n}=3)$ & $16.4 \pm 1.1$ & $34.3 \pm 22.5$ & $129.9 \pm 100.2$ & $3.6 \pm 0.9$ \\
\hline
\end{tabular}

Table 1. Insulin concentrations (pM) in culture medium supernatant. Note: all values are presented as the mean \pm S.D.

$\mathrm{Zn}^{2+}$ is an important metal ion related to a variety of metabolic functions. For example, DNA and RNA polymerases, which are necessary for cell proliferation, gene expression, and matrix metalloproteases, which operate in cell migration and invasion, are $\mathrm{Zn}^{2+}$-dependent enzymes ${ }^{23-25}$.

It has been hypothesised that $\mathrm{Zn}^{2+}$ might be absorbed for cell activity during differentiation and maturation, and secreted with insulin upon maturation. Therefore, $\mathrm{Zn}^{2+}$ dynamics in differentiated cells are considered as the novel marker for the maturation of generated IPCs.

Here, we determined whether the $\mathrm{Zn}^{2+}$ concentration in culture medium is an easy and useful marker of IPC differentiation and maturation, and demonstrate the mechanism of $\mathrm{Zn}^{2+}$ dynamics of IPCs.

\section{Results}

IPCs in 3D culture form cell clusters more easily than in conventional 2D culture. During twostep differentiation, there were some morphological differences between the conventional $2 \mathrm{D}$ culture and $3 \mathrm{D}$ culture due to the culture duration. In detail, in the 3D culture, ADSCs and RCP pieces gathered and underwent sphere-like formation within 24 hours of culture (Fig. 1A). Subsequently, IPCs generated in 3D culture exhibited sphere-like formations until 21 days, whereas cell clusters had formed at around day 21 in conventional 2D culture (Fig. 1B). Immunofluorescence staining detected human insulin in the cytoplasm of 3D-cultured IPCs on day 21 (Fig. 1C). These culture method-related differences revealed that the stimulation index (SI) was significantly higher in 3D-cultured IPCs compared with conventional 2D culture on day 21 (3.64 \pm 0.86 vs. $1.21 \pm 0.11$, $P<0.05$, Welch's test, Fig. 1D). Measured insulin concentrations including under the non-stimulated (basal) condition of each culture system are shown in Table 1.

3D-cultured IPCs reduce blood glucose levels of DM mice after transplantation. As an in vivo functional assay, after transplantation of $963 \mathrm{D}$-cultured IPCs into the mesentery of STZ-induced DM nude mice $(\mathrm{n}=4)$, blood glucose levels gradually decreased below $200 \mathrm{mg} / \mathrm{dl}$. Briefly, at 6 days after transplantation, the 
A
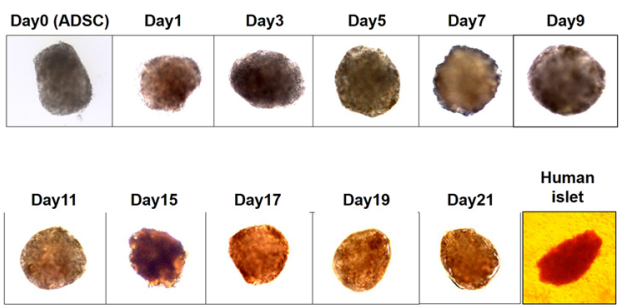

C

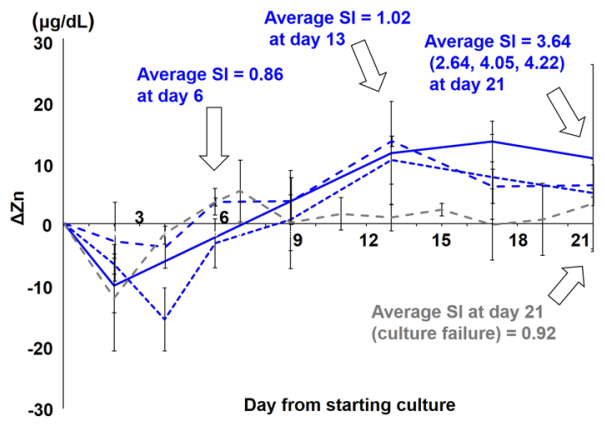

B

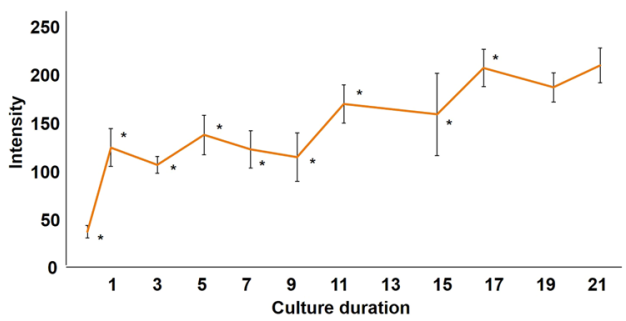

Figure 2. (A) Sphere-like formations containing ADSCs were gradually positively stained by dithizone every 2 days during culture. Representative cell formations $(n=8$ each time point) are shown. Freshly isolated human islets were included as a positive control (lower right corner). (B) Dithizone-stained cells were analysed by Image J. The staining intensity increased gradually and reached the peak on day 17 . The average of three independent experiments is shown. $* P<0.01$, Bonferroni's test. Error bars represent the standard deviation. (C) Pattern of $\mathrm{Zn}^{2+}$ concentration changes in culture supernatants under $3 \mathrm{D}$ culture conditions. $\Delta \mathrm{Zn}^{2+}=\mathrm{Zn}^{2+}$ (supernatant) $-\mathrm{Zn}^{2+}$ (fresh culture medium). Three independent experiments are shown as blue lines. Average SI at day 6, day 13 and day 21 are shown. Error bars represent the standard deviation. Grey-dotted line shows when cell culture failed to differentiate and mature properly because of the culture conditions. Average SI at day 21 was showed. Error bars represent the standard deviation.

blood glucose levels of four mice decreased to under $200 \mathrm{mg} / \mathrm{dl}$ and were maintained below this level until 30 days after transplantation $(4 / 4,100 \%)$, whereas the sham group $(n=4)$ could not convert their hyperglycaemic state to a normoglycaemia level (Fig. 1E).

3D-cultured IPCs contain secretory granules and secrete insulin. Electron microscopy showed insulin secretory granule-like structures and dense structures in 3D-cultured IPCs on day 21 as observed in human naïve $\beta$-cells as the control (Fig. $1 \mathrm{~F}$ ).

mRNA expression of differentiation marker genes in 3D-cultured IPCs. Expression of SOX17 as an endoderm development marker, of NGN3 as an endocrine cell differentiation marker, and of MAFA as an index of mature pancreatic $\beta$-cells, on day 17 were significantly higher compared with days 0 (SOX17, $P<0.01$; NGN3 and MAFA, $P<0.001$, Bonferroni's test, Fig. $1 G$ ) and day 7 (SOX17, $P<0.01$; NGN3 and MAFA, $P<0.001$, Bonferroni’s test, Fig. 1G).

Immunohistochemistry of IPCs showed strong insulin expression. To clarify the expression of insulin, IPCs were investigated using immunohistochemistry. The cytoplasm of IPCs in day $213 \mathrm{D}$ cultures stained strongly (especially granules-like structures) for insulin (Fig. 1H).

Dithizone stains IPCs gradually over time. In the 3D culture, the overall appearance of sphere-like formations was gradually stained by dithizone (Fig. 2A, eight IPCs observed at each point). Representative images of four independent experiments are shown in Fig. 2A. Quantification of images of cultured cells by Image ${ }^{26}$ showed that the staining intensity was gradually increased up to 180 and had statistically peaked on day $17(P<0.01$, Bonferroni's test) Based on three random points of eight IPCs, the average value is shown in Fig. 2B. After day 17, there were statistically no significant differences in the staining intensity of dithizone. As a reference, we evaluated the intensity in images of isolated human islets (Fig. 2A, right corner of the lower row), which was 244.

$\mathrm{Zn}^{2+}$ concentrations in culture medium change from negative to positive during proper IPC differentiation. $\quad \mathrm{Zn}^{2+}$ concentration changes in the culture supernatants $\left[\Delta \mathrm{Zn}^{2+}:\right.$ calculated as $\mathrm{Zn}^{2+}$ (supernatant) $-\mathrm{Zn}^{2+}$ (fresh culture medium) $]$ of the 3D-cultured IPCs were measured and plotted. In the early period 
A
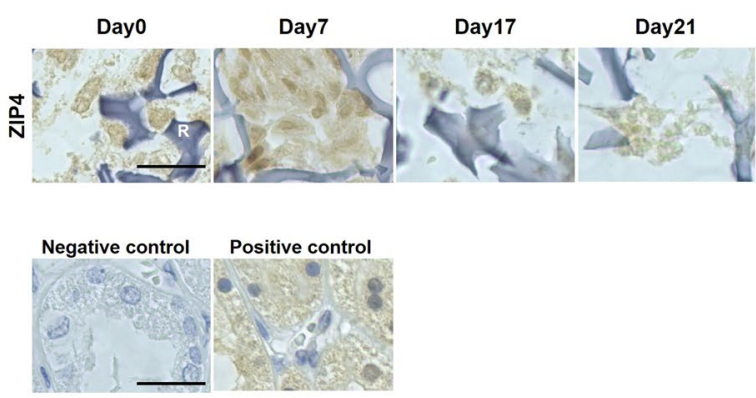

C
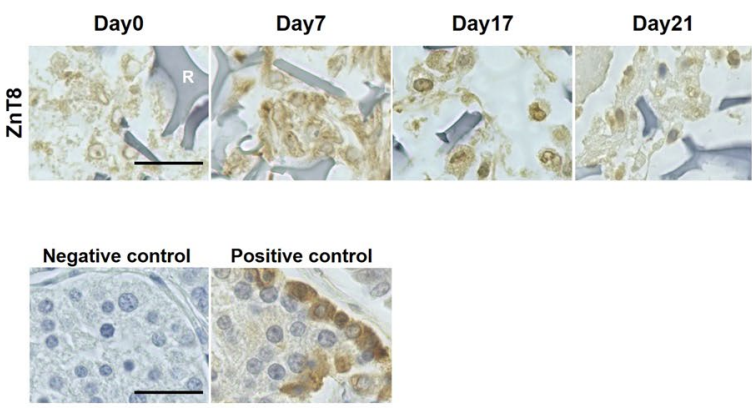

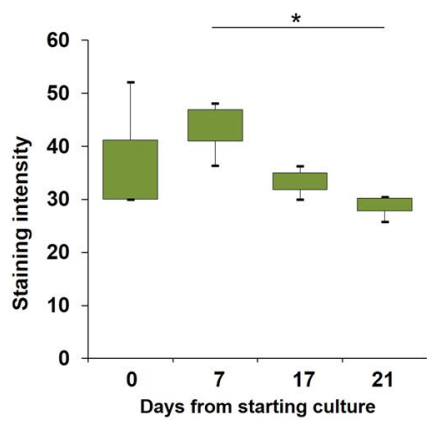

D

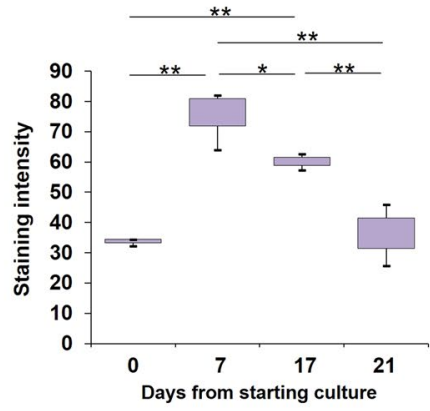

Figure 3. (A) Immunohistochemistry of ZIP4 at days $0,7,17$, and 21. Representative results from three independent experiments are shown. (B) Staining intensities in images were measured by ImageJ. (C) Immunohistochemistry of ZnT8 at days $0,7,17$, and 21. Representative results from three independent experiments are shown. (D) Staining intensities in images were measured by ImageJ. Three random fields were analysed. R: RCP petaloid $\mu$-piece, $* P<0.05$, $* * P<0.01$, Fisher's least significant difference. Error bars represent the standard deviation.

of days 3 and $6, \Delta \mathrm{Zn}^{2+}$ had a negative value. However, $\Delta \mathrm{Zn}^{2+}$ because positive from day 6 to 9 and reached its peak at day 13-17 (Blue lines in Fig. 2C, three independent experiments, SIs of each experiment were 2.64, 4.05, and 4.22, and the average SI was 3.64 at day $21,0.86$ at day 6 , and 1.02 at day 13 ). However, if we failed to differentiate and properly mature cells because of a lack of supplements, the increase of $\Delta \mathrm{Zn}^{2+}$ was low (grey-dotted line, Fig. 2C, average SI was 0.92 at day 21).

Zinc transporter expression in the early phase of the IPC differentiation protocol. ZIP4 expression was detected throughout the protocol, but reached its peak on day 7 and then decreased gradually $(\mathrm{P}<0.05$, vs day 21, Fisher's least significant difference, Fig. 3A,B). However, ZnT8 expression was strongly detected from day 7 and then decreased significantly $(P<0.01$, vs each point. $\mathrm{P}<0.05$, vs day 17 , Fisher's least significant difference, Fig. 3C,D).

Positive change pattern of the $\mathrm{Zn}^{2+}$ concentration might be specific, but a negative change pattern might be general in cells differentiated from ADSCs. $\Delta \mathrm{Zn}^{2+}$ of $3 \mathrm{D}$-cultured IPCs reached its peak at day 13-17 as described above. However, $\Delta \mathrm{Zn}^{2+}$ in the conventional $2 \mathrm{D}$ culture had an obviously different pattern from that in the 3D culture, especially in the positive curve area (Fig. 4A). In the 3D culture, an increase of $\Delta \mathrm{Zn}^{2+}$ during day 7-21 was statistically correlated with the quantified intensity of dithizone staining $\left(\mathrm{r}=0.798\right.$, Spearman's rank correlation coefficient). To estimate the meaning of the negative curve area, the $\mathrm{Zn}^{2+}$ concentration changes were measured in the supernatant of hepatocyte-like cells (HLCs) cultured in differentiation culture medium. Interestingly, the $\mathrm{Zn}^{2+}$ concentration change of culture supernatant in in early stage of the HLC differentiation protocol was also negative, and the positive curve pattern was different from that of IPCs in $3 \mathrm{D}$ and $2 \mathrm{D}$ cultures (Fig. 4B).

\section{Discussion}

Islet transplantation is a $\beta$-cell replacement therapy that has the potential to make type 1 diabetes mellitus (T1DM) patients free of insulin injections. However, islet transplantation is still not widespread because of some issues. One of them is the severe donor shortage in some countries such as Japan ${ }^{1,27}$. Moreover, repeat transplantation is often required to achieve an insulin-free status, which accelerates the donor shortage. To resolve this issue, it is desirable to establish more stable and safe cell sources. Therefore, insulin-producing cells (IPCs) derived from mesenchymal stem cells are expected to be an alternative and non-cadaveric therapeutic cell source. 
A

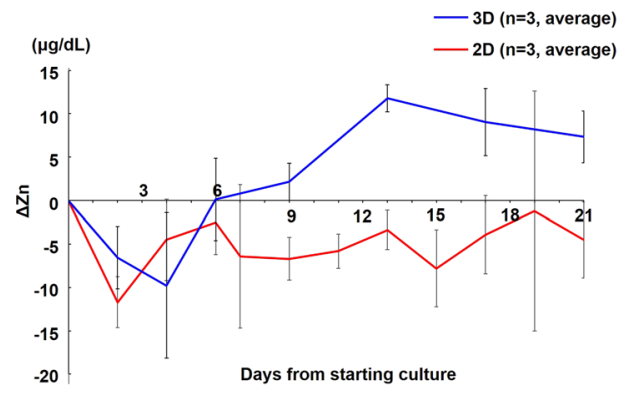

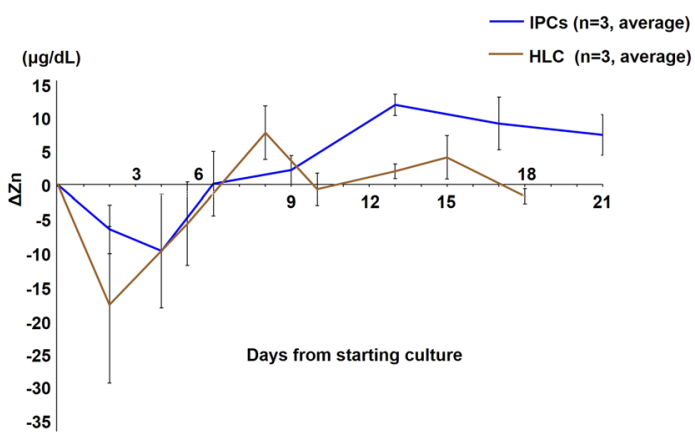

Figure 4. (A) Comparison of the pattern of $\mathrm{Zn}^{2+}$ concentration changes in the culture supernatant of IPCs $(\mathrm{n}=8$ at each point) under $3 \mathrm{D}$ culture and $2 \mathrm{D}$ culture conditions, $(\mathrm{B})$ or hepatocyte-like cell (HLC, $\mathrm{n}=8$ at each point) differentiation. Three independent experiments were performed. Error bars represent the standard deviation.

For clinical application of IPCs, cells function should be evaluated by a non-destructive procedure before transplantation. In previous methods including dithizone staining, RT-PCR, immunofluorescence staining, and western blotting, large numbers of IPCs must be sacrificed. It is well known that $\mathrm{Zn}^{2+}$ plays a critical role in insulin release metabolism, and glucose stimulation induces $\mathrm{Zn}^{2+}$ release from islet cells ${ }^{28}$. Thus, we focused on the $\mathrm{Zn}^{2+}$ concentration because evaluation of the culture supernatant is a non-invasive method for cells. In this study, we found that the peak of dithizone staining intensity was day 17 and elevated differentiation marker expression. Interestingly, the staining intensity of generated IPCs approached that of human islets. However, as indicated by the SI, the generated IPCs had not completely achieved the same functions of human islets yet. Remarkably, $\Delta \mathrm{Zn}^{2+}$ did not reach statistical significance after day 17. Therefore, the maturation of IPCs might be adequate for transplantation earlier than day 21. Moreover, if we did not differentiate and mature IPCs properly because of the medium conditions, $\Delta \mathrm{Zn}^{2+}$ did not have a high enough positive value and a sufficient SI could not be achieved. These findings suggested that $\Delta \mathrm{Zn}^{2+}$ as an indicator of zinc ion dynamics in the culture medium reflected the IPC functional maturation status. Therefore, IPC transplantation on day 17 might produce interesting results.

However, the weekly increase in SI seemed to be different from that of zinc ion concentration in step 2. This observation suggested a discrepancy between the creation of insulin secretory granules and the acquisition of the ability to release insulin (ability to detect a change of glucose concentration), and this sensory ability might be obtained in the later phase of IPC differentiation.

Recently, $\mathrm{Zn}^{2+}$ transporters have been focused on for their roles in cells because $\mathrm{Zn}^{2+}$ is a functional component in many cellular proteins and enzymes, and plays a key role in cell growth and development ${ }^{29-31}$. $\mathrm{Zn}^{2+}$ transporters include the zinc transporter protein (ZnT)/solute carrier 30A (SLC30A) family and Zrt, Irt-like protein (ZIP)/SLC39A family. Many reports indicate that the $\mathrm{ZnT}$ family carries $\mathrm{Zn}^{2+}$ from the cytoplasm to organelles or extracellular regions, while the ZIP family carries $\mathrm{Zn}^{2+}$ retrodirectionally ${ }^{21,30-32}$. Among them, ZnT8 expression is detected mainly in insulin secretory granules ${ }^{33-35}$. Some T1DM cases are caused by an autoimmune disorder against $\mathrm{ZnT} 8^{36-41}$. In a recent study, ZnT8 expression was correlated with insulin secretion in rat islet cell line INS-1E $\mathrm{E}^{42}$. In addition, it has also been reported that ZIP4 expression is associated with intake of $\mathrm{Zn}^{2+}$ by islet $\beta$ cells $^{43}$ and their differentiation and maturation ${ }^{44,45}$. In previous reports, stem cells have absorbed $\mathrm{Zn}^{2+}$ in their nuclei during differentiation ${ }^{46,47}$. In HLCs derived from ADSCs, we showed that $\Delta \mathrm{Zn}^{2+}$ values were negative in the early period of differentiation. This result suggest that ADSCs require a large amount of $\mathrm{Zn}^{2+}$ in the early stage of differentiation, at least for differentiation into endodermal cells, which is probably used to generate organelles and synthesise $\mathrm{RNA}^{47,48}$. Moreover, $\mathrm{Zn}^{2+}$ absorption appears to occur in embryonic stem cells during endoderm transition ${ }^{46,48}$. Therefore, based on our results above and previous reports, we predicted the mechanism of $\mathrm{Zn}^{2+}$ changes during IPC differentiation (Fig. 5). Briefly, ADSCs start to generate insulin secretory granules after induction to endoderm and cell fate decision. To achieve these events, a large degree of RNA synthesis is required. Thus, ZIP4 performs an essential role for cell development ${ }^{44,45}$, which we named the "RNA synthesis and organelle generation phase" (Fig. 5 left). Then, along with increases of secretory granules, which contain insulin hexamers with two $\mathrm{Zn}^{2+}$ and $\mathrm{ZnT} 8^{34,35}$, the intensity of dithizone staining increases. After sufficient maturation, many secretory granules containing insulin and $\mathrm{Zn}^{2+}$ are gradually released from IPCs, resulting in an increase of the $\mathrm{Zn}^{2+}$ concentration, which we named the "secretory phase".

Regarding the limitations of this study, there are some issues to be investigated further. First, essentially, there are no fixed evaluation methods for IPC differentiation and maturation. Thus, it is still unclear whether these IPCs differentiate and mature sufficiently. Second, in our study, marker gene expression was evaluated by RT-PCR only. Therefore, marker gene expressions should be confirmed by western blotting and immunostaining. Similarly, zinc transporter protein expression was measured by immunohistochemistry only. By performing further experiments, the results of this study may confirm our hypothesis (Fig. 5). Third, during the differentiation of stem cells, further analyses are required to understand zinc ion dynamics. According to our results from HLCs as another cell type, at least ADSCs require a large amount of $\mathrm{Zn}^{2+}$ to convert into cells of other germ 
RNA synthesis and organelle generation phase
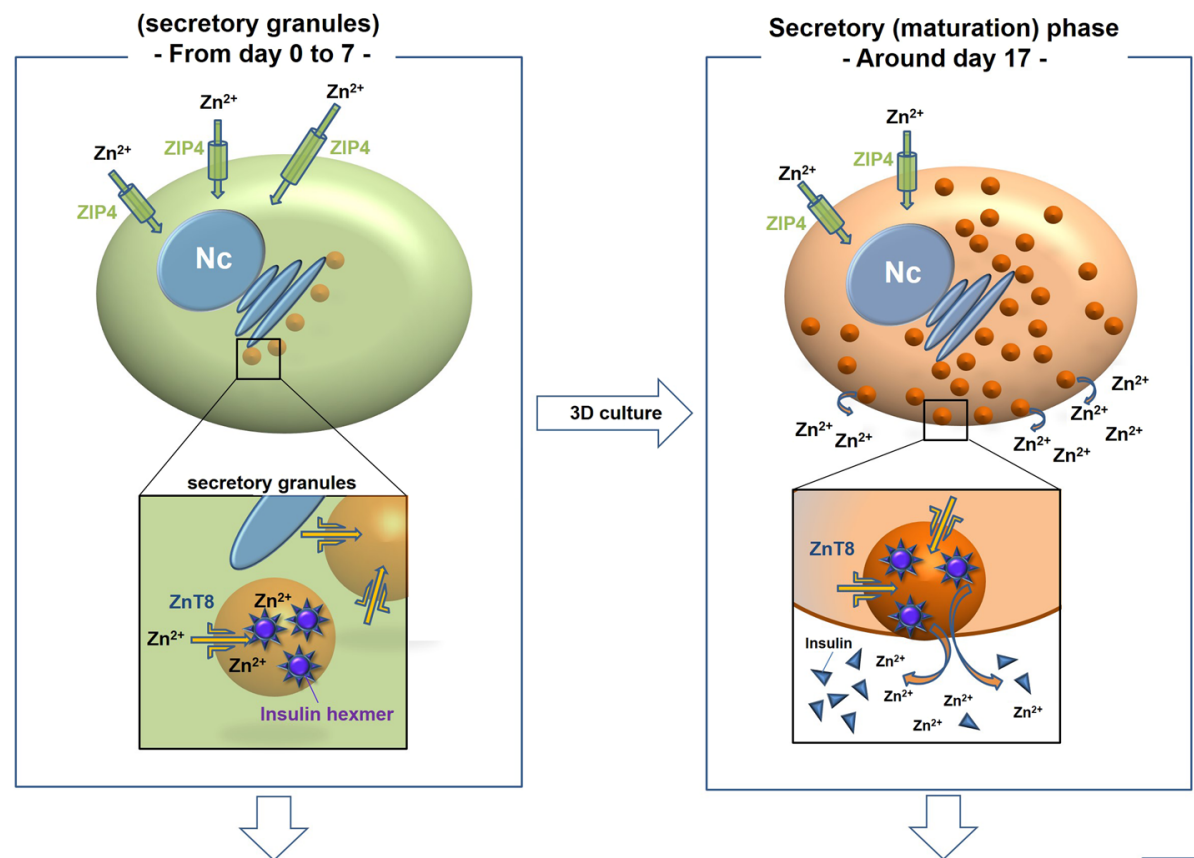

Zn balance: negative

$\mathrm{Zn}^{2+}$ intake

(synthesis of RNA, generating granules)
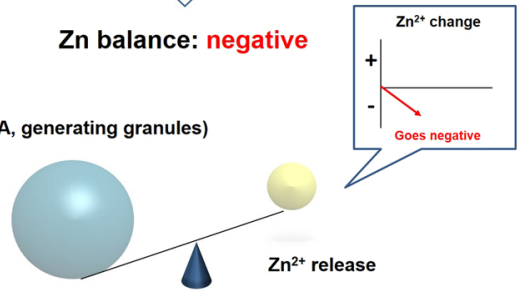

$\checkmark$ Cells requires $\mathrm{Zn}^{2+}$ for $\mathrm{RNA}$ synthesis and organelle generation

$\checkmark$ ZIP4 expression $\uparrow$

$\checkmark \mathrm{Zn}^{2+}$ absorption $\uparrow$

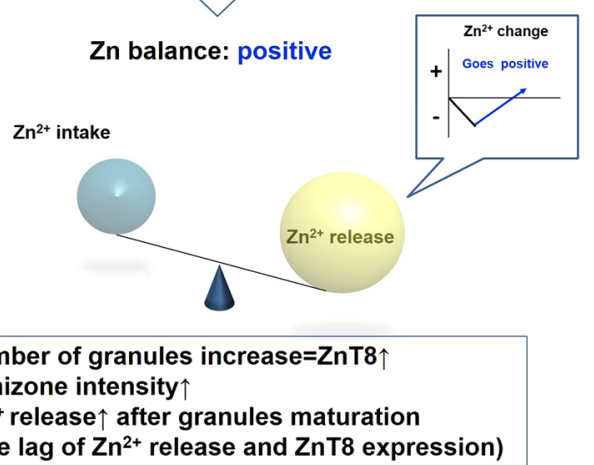

Figure 5. ADSC started to generate insulin secretory granules after induction to endoderm-derived cells and fate decision. To achieve these events, mass RNA synthesis is required. Thus, it was considered that ZIP4 was expressed and performed an essential role in cell development ("RNA synthesis and organelle generation phase", left). Then, along with increases of secretory granules containing insulin hexamers with two $\mathrm{Zn}^{2+}$ each and expression of $\mathrm{ZnT}$, the intensity of dithizone staining was increased. After sufficient maturation, many secretory granules containing insulin and $\mathrm{Zn}^{2+}$ were gradually released from IPCs. Such release resulted in an increase of the $\mathrm{Zn}^{2+}$ concentration ("Secretory phase", right).

layers such as endoderm and generate organelles. Zinc is essential and required for cell differentiation ${ }^{44,45}$. Thus, zinc ion dynamics might reflect cell fate decisions. Therefore, evaluating $\mathrm{Zn}^{2+}$ dynamics in the differentiation of other germ layer cells might support our hypothesis (Fig. 5). Fourth, the precise microstructures of IPCs are still unknown. We observed some undefined "empty granules" in 3D-generated IPCs by electron microscopy; however, further investigations are required to determine whether these structures are special IPCs structures or generated as a result of cell preparation or storage, or cell damage.

In conclusion, $\mathrm{Zn}^{2+}$ concentration changes in the culture supernatant might be a marker of IPC maturation. This new insight might be useful in validating the function of transplantable IPCs, excluding inadequate IPCs for transplantation, or monitoring production.

Materials and Methods

ADSC preparation. STEMPRO ${ }^{\mathrm{TM}}$ Human Adipose-Derived Stem Cells (Invitrogen, Grand Island, NY) were used in this study. ADSCs were cultured in ADSC basal medium consisting of MesenPROTM RS (Gibco, Carlsbad, CA) with GlutaMAXTM-I (Gibco).

IPC differentiation protocol. IPCs were generated as reported previously ${ }^{16,17}$. Briefly, after sufficient passaging, ADSCs were mixed with recombinant peptide micro-pieces (RCP $\mu$-pieces; FUJIFILM, Tokyo, Japan) and transferred to a Nunchlon Sphera 96U Bottom Plate (Thermo Scientific, Waltham, MA) for 3D culture. For conventional 2D culture, ADSCs without RCP $\mu$-pieces were seeded into FALCON ${ }^{\mathrm{TM}} 6$ Well Flat Bottom Tissue Culture Plate with a Low Evaporation Lid (Corning Co., Corning, NY). The protocol for IPC induction has 
been reported previously ${ }^{18}$. However, the contents of the differentiation cocktail were changed to recombinant human activin-A, hepatocyte growth factor, and albumin. ADSCs were cultured using the step-1 (from day 0 to 7) medium consisting of Dulbecco's modified Eagle's medium/F12 (Gibco) with 1\% recombinant human albumin (Wako, Osaka, Japan), $10 \mathrm{nM}$ exendin-4 (Sigma-Aldrich, St. Louis, MO), 1\% N2 supplements (Gibco), 1\% B27 supplement (Gibco), and $50 \mathrm{ng} / \mathrm{mL}$ recombinant human activin-A (PeproTech, Rocky Hill, NJ). Step-2 (from day 8 to 21) medium was the same as step-1 medium with addition of $50 \mathrm{ng} / \mathrm{mL}$ recombinant human hepatocyte growth factor (PeproTech), valproic acid (Wako) and $10 \mathrm{mM}$ nicotinamide (Sigma-Aldrich). The culture medium was changed and the supernatant was collected every 2 days.

Hepatocyte-like cell differentiation protocol. Hepatocyte-like cells (HLCs) were differentiated as reported previously ${ }^{49}$. Briefly, ADSCs were cultured using a 20-day differentiation protocol under 2D culture conditions. The differentiation protocol employed $2 \mu \mathrm{M}$ Chir99021 (MedChemExpress, Monmouth Junction, NJ, USA), 1\% ITS (Sigma-Aldrich), $20 \mathrm{ng} / \mathrm{mL}$ BMP2 (PeproTech), $30 \mathrm{ng} / \mathrm{mL}$ FGF4 (PeproTech), $20 \mathrm{ng} / \mathrm{mL}$ HGF (PeproTech), $10 \mathrm{ng} / \mathrm{mL}$ oncostatin M (PeproTech), and $10 \mu \mathrm{M}$ dexamethasone (Sigma-Aldrich). Eight HLCs were investigated at each time point. Three independent experiments were performed for the cell analysis.

Glucose-stimulated insulin secretion test. A glucose-stimulated insulin secretion test was performed according to previous reports ${ }^{50-52}$. Briefly, differentiated cells were stimulated by 2.2 or $22 \mathrm{mM}$ glucose in Krebs-Ringer's solution for 1 hour at $37^{\circ} \mathrm{C}$ in a $5 \% \mathrm{CO}_{2}$ incubator. Krebs-Ringer's solution consisted of $129 \mathrm{mM}$ $\mathrm{NaCl}, 1.2 \mathrm{mM} \mathrm{MgSO}_{4}, 1.2 \mathrm{mM} \mathrm{KH}_{2} \mathrm{PO}_{4}, 4.7 \mathrm{mM} \mathrm{KCl}, 5 \mathrm{mM} \mathrm{NaHCO}_{3}, 2.5 \mathrm{mM} \mathrm{CaCl}_{2}, 10 \mathrm{mM} \mathrm{HEPES}_{\text {, and } 1 \%}$ bovine serum albumin (Sigma-Aldrich). After stimulation, the supernatant of each sample was collected, and human insulin concentrations were measured by a Human/Canine/Porcine Insulin Quantikine ELISA Kit (R\&D Systems, Minneapolis, MN). The optical absorbance was measured by a SpectraMax i3 (Molecular Devices, Sun Jose, CA) and SoftMax Pro 7 (Molecular Devices). The stimulation index (SI) was calculated as the ratio of the insulin measurement after $22 \mathrm{mM}$ glucose stimulation divided by the insulin measurement after $2.2 \mathrm{mM}$ glucose stimulation. Three independent experiments were performed to evaluate GSIS $(n=3)$.

Electron microscopy. Cells were prepared as reported previously ${ }^{17}$. Briefly, 3D-cultured IPCs were fixed in $0.1 \mathrm{M}$ phosphate buffer with $2 \%$ paraformaldehyde and $2 \%$ glutaraldehyde at $4{ }^{\circ} \mathrm{C}$ overnight on day 21 . After dehydration in graded ethanol solutions, the IPC samples were infiltrated by propylene oxide and resin (Nissin EM, Tokyo, Japan) for 1 hour. After transfer to fresh $100 \%$ resin, the IPC samples were polymerised at $60^{\circ} \mathrm{C}$ for 48 hours and then sectioned at $70 \mathrm{~nm}$ thicknesses using an ultramicrotome (Leica, Vienna, Austria). The sections were stained with $2 \%$ uranyl acetate at room temperature for 15 minutes. After washing with distilled water, the samples were secondary stained with a Lead stain solution (Sigma-Aldrich) at room temperature for 3 minutes. Electron microscopy was performed at Tokai Electron Microscopy Inc. (Nagoya, Japan). Sections were observed under a JEM-1400 Plus transmission electron microscope (JEOL Ltd. Tokyo, Japan) at an acceleration voltage of $100 \mathrm{kV}$. Images were obtained by an EM-14830RUBY2 CCD camera (JEOL Ltd). As a control, we have included a human $\beta$-cell image obtained by electron microscopy (Fig. 1F, right, this image was reused in this study under an agreement with Elsevier ${ }^{25}$ ).

Dithizone staining. Cultured cells ( $\mathrm{n}=8$, each day of the evaluation) were stained by a dithizone solution. The dithizone solution consisted of $50 \mathrm{mg}$ dithizone (Wako) per $5 \mathrm{~mL}$ dimethyl sulfoxide (Wako). The IPCs were incubated in the dithizone solution at $37^{\circ} \mathrm{C}$ with $5 \% \mathrm{CO}_{2}$ after washing with PBS three times. The stained samples were assessed by a BZ-X710 multi-purpose microscope (KEYENCE Engineering, Tokyo, Japan) and BZ-X Analyzer (KEYENCE Software, Japan).

$\mathrm{Zn}^{2+}$ concentration assay. The culture supernatant was collected during medium changes. The $\mathrm{Zn}^{2+}$ concentration in each IPC sample was evaluated by a Metallo Assay kit zinc LS (Metallogenics Co., Chiba, Japan), according to manufacturer's protocol. Briefly, $230 \mu \mathrm{l}$ buffer, $12 \mu \mathrm{l}$ sample, and $5 \mu \mathrm{l}$ chelate colour solution were mixed and then transferred to a clear 96-well flat bottom plate (Corning Co.). Sample plates were analysed at $560 \mathrm{~nm}$ optical absorbance. The $\mathrm{Zn}^{2+}$ concentration was determined according to the optical absorbance of the standard sample $(200 \mathrm{mg} / \mathrm{dl})$ and blank sample (deionised distilled water). Optical absorbance were measured by a SpectraMax i3 (Molecular Devices) and SoftMax Pro 7 (Molecular Devices). Each Zn2+ concentration change was calculated as: $\Delta \mathrm{Zn}^{2+}=\mathrm{Zn}^{2+}$ (supernatant) $-\mathrm{Zn}^{2+}$ (fresh culture medium). Eight IPCs were investigated at each time point. Three independent experiments were performed for this assay $(n=3)$.

Immunohistochemical staining. IPC samples were fixed in formalin overnight and embedded in paraffin after embedding in iPGell (Genostaff, Tokyo, Japan). Four micrometre-thick sections of IPC samples and human kidney and pancreas (renal tubule cells and islets as negative and positive controls, respectively) were prepared. Usage of these resected specimens was approved by Tokushima University Hospital (Tokushima Clinical Trial Management System 2900-1, Dec 25, 2017). Specimen collection was performed in accordance with relevant guidelines/regulations. All patients had provided written informed consents. Sections were dewaxed, deparaffinised in xylene (Wako), and rehydrated through a series of graded alcohol solutions. Endogenous peroxidases were blocked with $0.3 \%$ hydrogen peroxidase (Wako) for 20 minutes. For antigen retrieval, the sections were boiled in EDTA buffer ( $\mathrm{pH}$ 6.0) using a microwave for 15 minutes at $1400 \mathrm{~W}$ and then for 10 minutes at $700 \mathrm{~W}$. The sections were incubated in Protein Serum Free (Dako, Burlington, Canada) for 10 minutes to prevent nonspecific antigen binding. Samples were incubated with primary antibodies for 1.5 hours at room temperature. Primary antibodies were: anti-insulin (aa287-299, LS-B129; 1:100, LSBio, Seattle, WA), anti-human ZnT8 (16169-1-AP, 1:50, Proteintech, Chicago, IL) and anti-human ZIP4 (20625-1-AP, 1:200, Proteintech). The sections were then 
incubated with a secondary antibody (EnVision Dual Link System-HRP, DAKO) for 1 hour at room temperature. Sections were developed in diaminobenzidine (Wako) and counterstained with Mayer's haematoxylin (Muto pure chemicals, Tokyo, Japan). The stained sections were dehydrated in graded alcohol solutions and coverslipped.

Immunofluorescence staining. Specimens of IPCs were sectioned, dewaxed, deparaffinised, and rehydrated. Blocking of endogenous peroxidases and retrieval of antigens were performed as described above. To prevent nonspecific antigen binding, the sections were incubated in 3\% BSA for 1 hour. The sections were then incubated with an anti-human insulin antibody (\#4590S, Cell Signaling Technology, Danvers, MA) as the primary antibody at $4{ }^{\circ} \mathrm{C}$ overnight. The sections were incubated with a secondary antibody, Alexa Fluor 555 goat anti-rabbit IgG (A-21244, 1:500, Invitrogen), after washing with PBS three times. Following washes with PBS, the sections were incubated with $4^{\prime}$,6-diamidino-2-phenylindole (28718-90-3, 1:2000, Santa Cruz Biotechnology, Paso Robles, CA) to detect nuclei. The stained samples were washed with PBS and coverslipped.

Quantitative imaging analysis. Images of cells were digitally analysed by ImageJ (National Institute of Health, Bethesda, ML) ${ }^{26}$. In the analysis of dithizone staining, images of cells were converted to hue, saturation and value (HSV) models and filtered by hue thresholds of 7-20. The mean grey value of saturation of three random points in one spheroid area was measured in filtered images as the staining intensity ${ }^{52}$. In the analysis of immunohistochemical staining, after divided images into three channels, the images were filtered by hue thresholds of 20-28. The mean grey value of saturation of three random points in one spheroid area and background were measured in filtered images. The staining intensity of immunohistochemistry was calculated as the ratio of the mean grey value in the spheroid area divided by that of the background. Staining intensity data were calculated as the average of eight cell spheres (three random points of IPCs, $\mathrm{n}=8$ ).

IPC transplantation into streptozotocin-induced diabetic nude mice. As an in vivo functional assay, transplantation of IPCs was undertaken as described in our previous report ${ }^{17}$. Briefly, $200 \mathrm{mg} / \mathrm{kg}$ body weight streptozotocin (STZ; Sigma-Aldrich) was dissolved in citrate buffer ( $\mathrm{pH} 4.5$ ) and administered to 5-6 week-old BALB/c nude mice (Charles River Japan, Yokohama, Japan) by intraperitoneal injection. STZ-induced DM nude mice were defined as those with blood glucose values over $350 \mathrm{mg} / \mathrm{dl}$ for two continuous readings or $400 \mathrm{mg} / \mathrm{dl}$ in one reading. Ninety-six IPCs were transplanted into the mesentery of STZ-induced DM mice, according to our previous method for IPC transplantation ${ }^{17}$. Sham mouse group mice $(\mathrm{n}=4$, administered normal saline intra-mesentery) and naïve nude mouse group $(n=4)$ were included. After transplantation, blood glucose values obtained using a Medisafe Fit kit (TERUMO, Tokyo, Japan) and body weight were recorded every 2 days, including sham and naïve nude mouse groups. All mice were bred in the animal facility at Tokushima University. Experiments and procedures were approved by the Animal Care and Use Committee of Tokushima University and performed in accordance with the NIH Guide for the Care and Use of Laboratory Animals.

Quantitative reverse transcription-polymerase chain reaction analyses. Total RNA was extracted from cultured cells with an RNeasy Mini Kit (QIAGEN, Hilden, Germany), according to the manufacturer's instructions. RNA purity was assessed using a Nano Drop ND-1000 spectrometer (Thermo Scientific). cDNA was synthesised from total RNA using a reverse transcription kit (QIAGEN). Quantitative reverse transcription-polymerase chain reaction analysis was performed using TaqMan Gene Expression Assays in 7500 real-time polymerase chain reaction system with StepOne Plus software (Applied Biosystems, Foster City, CA). The gene expression level was normalised to the GAPDH gene expression level. Primers for SOX17 (Hs00751752_s1, Applied Biosystems) and NGN3 (Hs01875204_s1, Applied Biosystems) were used in this study.

Statistical analysis. Statistical calculations were performed using SPSS statistics version 24 (IBM, Chicago, IL) or State Mate III (ATMS Co., LTD. Tokyo, Japan). Sample data were compared by the Mann-Whitney U-test, Student's and Welch's t-tests, or chi-squared test. Comparisons between multiple groups were performed by one-way ANOVA, Bonferroni's test, and Fisher's least significant difference. P-values of less than 0.05 were considered as significant.

\section{Data availability}

The datasets used and/or analysed in this study are available from the corresponding author upon reasonable request.

Received: 18 July 2019; Accepted: 25 November 2019;

Published online: 10 December 2019

\section{References}

1. Pellegrini, S., Piemonti, L. \& Sordi, V. Pluripotent stem cell replacement approaches to treat type 1 diabetes. Curr Opin Pharmacol. 43, 20-26 (2018)

2. D’Amour, K. A. et al. Production of pancreatic hormone-expressing endocrine cells from human embryonic stem cells. Nat Biotechnol. 24, 1392-1401 (2006).

3. Kroon, E. et al. Pancreatic endoderm derived from human embryonic stem cells generates glucose-responsive insulin-secreting cells in vivo. Nat Biotechnol. 26, 443-452 (2008)

4. Vegas, A. J. et al. Long-term glycemic control using polymer-encapsulated human stem cell-derived beta cells in immune-competent mice. Nat Med. 22, 306-311 (2016).

5. Kassem, D. H. \& Kamal, M. M. El-Kholy, Ael-L. and El-Masallamy, H.O. Exendin-4 enhances the differentiation of Wharton's jelly mesenchymal stem cells into insulin-producing cells through activation of various $\beta$-cell markers. Stem Cell Res Ther. 7, 108 (2016).

6. Santamaria, X., Massasa, E. E., Feng, Y., Wolff, E. \& Taylor, H. S. Derivation of insulin producing cells from human endometrial stromal stem cells and use in the treatment of murine diabetes. Mol Ther. 19, 2065-2071 (2011). 
7. Lemper, M. et al. Reprogramming of human pancreatic exocrine cells to $\beta$-like cells. Cell Death Differ. 22, 117-130 (2015).

8. Xin, Y. et al. Insulin-Producing Cells Differentiated from Human Bone Marrow Mesenchymal Stem Cells In Vitro Ameliorate Streptozotocin-Induced Diabetic Hyperglycemia. PLoS One. 12, 11 (2016).

9. Sapir, T. et al. Cell-replacement therapy for diabetes: Generating functional insulin-producing tissue from adult human liver cells. Proc Natl Acad Sci USA 102, 7964-7969 (2005).

10. Pagliuca, F. W. et al. Generation of functional human pancreatic $\beta$ cells in vitro. Cell. 159, 428-439 (2014).

11. Shiroi, A. et al. Identification of insulin-producing cells derived from embryonic stem cells by zinc-chelating dithizone. Stem Cells. 20, 284-292 (2009).

12. Saito, Y. et al. Homing effect of adipose-derived stem cells to the injured liver: the shift of stromal cell-derived factor 1 expressions. J Hepatobiliary Pancreat Sci. 21, 873-880 (2014).

13. Oedayrajsingh-Varma, M. J. et al. Adipose tissue-derived mesenchymal stem cell yield and growth characteristics are affected by the tissue-harvesting procedure. Cytotherapy. 8, 166-177 (2006).

14. Saito, Y. et al. The protective effect of adipose-derived stem cells against liver injury by trophic molecules. J Surg Res. 180, 162-168 (2013).

15. Utsunomiya, T. et al. Human adipose-derived stem cells: potential clinical applications in surgery. Surg Today. 41, 18-23 (2011).

16. Ikemoto, T. et al. A New 2-Step Acceleration Protocol Using a Histone Deacetylase Inhibitor to Generate Insulin-Producing Cells From Adipose-Derived Mesenchymal Stem Cells. Pancreas. 47, 477-481 (2018).

17. Ikemoto, T. et al. In vitro and in vivo effects of insulin-producing cells generated by xeno-antigen free 3D culture with RCP piece. Sci rep. 9, 10759 (2019).

18. Schulz, T. C. et al. A scalable system for production of functional pancreatic progenitors from human embryonic stem cells. PLoS One. 7, e37004 (2012).

19. Zalewski, P. D. et al. Video image analysis of labile zinc in viable pancreatic islet cells using a specific fluorescent probe for zinc. J Histochem Cytochem. 42, 877-884 (1994).

20. Clark, S. A., Borland, K. M., Sherman, S. D., Rusack, T. C. \& Chick, W. L. Staining and in vitro toxicity of dithizone with canine, porcine, and bovine islets. Cell Transplant. 3, 299-306 (1994).

21. Li, Y. V. Zinc and insulin in pancreatic beta-cells. Endocrine. 45, 178-189 (2014).

22. Blundell, T. L. et al. Three-dimensional atomic structure of insulin and its relationship to activity. Diabetes. 21, 492-505 (1972).

23. Andreini, C., Banci, L., Bertini, I. \& Rosato, A. Counting the zinc-proteins encoded in the human genome. J Proteome Res. 5, 196-201 (2006).

24. Andreini, C. \& Bertini, I. A bioinformatics view of zinc enzymes. J Inorg Biochem. 111, 150-156 (2012).

25. Orci, L. et al. Proteolytic maturation of insulin is a post-Golgi event which occurs in acidifying clathrin-coated secretory vesicles. Cell. 19(49), 865-868 (1987).

26. Schneider, C. A., Rasband, W. S. \& Eliceiri, K. W. NIH Image to ImageJ: 25 years of image analysis. Nat Methods. 9, 671-675 (2012).

27. The Japanese Pancreas and Islet Transplantation Association. Islet transplantation in Japan -Report from Japanese Islet Transplantation Registry-. Jpn J Transplant 53, 149-156 (2018).

28. Slepchenko, K. G., James, C. B. \& Li, Y. V. Inhibitory effect of zinc on glucose-stimulated zinc/insulin secretion in an insulinsecreting $\beta$-cell line. Exp Physiol. 98, 1301-1311 (2018).

29. Maret, W. New perspectives of zinc coordination environments in proteins. J Inorg Biochem. 111, 110-116 (2012).

30. Eide, D. J. Zinc transporters and the cellular trafficking of zinc. Biochim Biophys Acta. 1763, 711-722 (2006).

31. Cousins, R. J., Liuzzi, J. P. \& Lichten, L. A. Mammalian zinc transport, trafficking, and signals. J Biol Chem. 281, 24085-24859 (2006).

32. Kambe, T., Yamaguchi-Iwai, Y., Sasaki, R. \& Nagao, M. Overview of mammalian zinc transporters. Cell Mol Life Sci. 61, 49-68 (2004).

33. Merriman, C., Huang, Q., Gu, W., Yu, L. \& Fu, D. A subclass of serum anti-ZnT8 antibodie directed to the surface of live panreatic B-cells. J Biol Chem. 293, 579-587 (2018).

34. Chimienti, F., Devergnas, S., Favier, A. \& Seve, M. Identification and cloning of a beta-cell-specific zinc transporter, ZnT-8, localized into insulin secretory granules. Diabetes. 53, 2330-2337 (2004).

35. Chimienti, F. et al. In vivo expression and functional characterization of the zinc transporter ZnT8 in glucose-induced insulin secretion. J Cell Sci. 119, 4199-4206 (2006).

36. Chujo, D. et al. ZnT8-Specific CD4+ T cells display distinct cytokine expression profiles between type 1 diabetes patients and healthy adults. PLoS One. 8, e55595 (2013).

37. Dang, M. et al. Human type 1 diabetes is associated with T cell autoimmunity to zinc transporter 8. J Immunol. 61, 49-68 (2011).

38. Enee, E. et al. ZnT8 is a major CD8+ T cell-recognized autoantigen in pediatric type 1 diabetes. Diabetes. 61, 1779-1784 (2012).

39. Hamel, Y. et al. A unique CD8(+) T lymphocyte signature in pediatric type 1 diabetes. J Autoimmun. 73, 54-63 (2016).

40. Yu, C. et al. Identification of Candidate Tolerogenic CD8(+) T Cell Epitopes for Therapy of Type 1 Diabetes in the NOD Mouse Model. J Diabetes Res. 2016, 9083103 (2016).

41. Wenzlau, J. M. et al. The cation efflux transporter ZnT8 (Slc30A8) is a major autoantigen in human type 1. diabetes. Proc Natl Adac Sci USA 104, 17040-17045 (2007).

42. Huang, Q., Merriman, C., Zhang, H. \& Fu, D. Coupling of Insulin Secretion and Display of a Granule-resident Zinc Transporter ZnT8 on the Surface of Pancreatic Beta Cells. J Biol Chem. 292, 4034-4043 (2017).

43. Hardy, A. B. et al. Zip4 mediated zinc influx stimulates insulin secretion in pancreatic beta cells. PLoS One. 25, e0119136 (2015).

44. Jou, M. Y., Philipps, A. F., Kelleher, S. L. \& Lönnerdal, B. Effects of zinc exposure on zinc transporter expression in human intestinal cells of varying maturity. J Pediatr Gastroenterol Nutr. 50, 587-595 (2010).

45. Gefeller, E. M. et al. Regulation of intracellular $\mathrm{Zn}$ homeostasis in two intestinal epithelial cell models at various maturation time points. J Physiol Sci. 65, 317-328 (2015).

46. Wolford, J. L. et al. Loss of pluripotency in human embrynic stem cells directly correlates with an increase in nuclear zinc. PLoS One. 5, e12308 (2010).

47. Kitamura, H. et al. Toll-like receptor-mediated regulation of zinc homeostasis influences dendritic cell function. Nat Immunol. 7 , 971-977 (2006).

48. Lu, J. et al. Single-cell RNA sequencing reveals metallothionein heterogeneity during hESC differentiation to definitive endoderm. Stem Cell Res. 28, 48-55 (2018).

49. Huang, J., Guo, X., Li, W. \& Zhang, H. Activation of Wnt/ $\beta$-catenin signalling via GSK3 inhibitors direct differentiation of human adipose stem cells into functional hepatocytes. Sci Rep. 17, 40716 (2017).

50. Hannan, N. R. et al. Generation of multipotent foregut stem cells from human pluripotent stem cells. Stem Cell Reports. 10, 293-306 (2013).

51. Maehr, R. et al. Generation of pluripotent stem cells from patients with type 1 diabetes. Proc Natl Acad Sci USA 106, 15768-15773 (2009).

52. Sakata, N., Egawa, S., Sumi, S. \& Unno, M. Optimization of glucose level to determine the stimulation index of isolated rat islets. Pancreas. 36, 417-423 (2008). 


\section{Acknowledgements}

We thank Dr. Shinichi Matsumoto who kindly provided the image of the freshly isolated human islets for image analysis and Dr. Hisanori Uehara who gave us useful suggestions for electron microscopic images. We also thank Dr. Yusuke Arakawa and Dr. Kazunori Tokuda for assistance with some experiments and assays, and B.S. Yumi Horikawa for technical support. We thank Edanz Group (www.edanzediting.com/ac) for editing a draft of this manuscript. Dr. Ikemoto and Elsevier consists of the license details and the terms and conditions provided by Elsevier and Copyright Clearance Center for the reuse of the published figure (Fig. 1F, right).

\section{Author contributions}

S.O. performed in vitro experiments, prepared the mice, analysed data, and wrote the draft of the manuscript. T.I., S.Y., Y.S., and S.I. performed transplantation experiments and conducted cell culture. T.I. and Y.M. performed in vivo experiments. Y.W. and S. Imura conducted in vitro ADSC experiments. Y.W. conducted cell culture and analysed cultured cells. S.O., Y.W., and T.I. analysed the characteristics and functions of IPCs and edited the manuscript; M.S. and T.I. conducted and checked the experiments and edited the manuscript. All authors reviewed the manuscript.

\section{Competing interests}

Dr. Ikemoto and Dr. Shimada were funded by the Regenerative Medicine Cooperative of Fujifilm Corporation and Tokushima University. Dr. Ikemoto, Dr. Yamada, Dr. Saito, Dr. Morine, and Dr. Imura were also funded in part by grants-in-aid for Scientific Research from the Ministry of Education, Science and Culture of Japan (grant no. 16K10429). Dr. Ikemoto and Dr. Shimada were also funded in part by a grant from the Japan IDDM network. Dr. Ohta and Dr. Wada declare no potential conflicts of interest.

\section{Additional information}

Correspondence and requests for materials should be addressed to T.I.

Reprints and permissions information is available at www.nature.com/reprints.

Publisher's note Springer Nature remains neutral with regard to jurisdictional claims in published maps and institutional affiliations.

Open Access This article is licensed under a Creative Commons Attribution 4.0 International License, which permits use, sharing, adaptation, distribution and reproduction in any medium or format, as long as you give appropriate credit to the original author(s) and the source, provide a link to the Creative Commons license, and indicate if changes were made. The images or other third party material in this article are included in the article's Creative Commons license, unless indicated otherwise in a credit line to the material. If material is not included in the article's Creative Commons license and your intended use is not permitted by statutory regulation or exceeds the permitted use, you will need to obtain permission directly from the copyright holder. To view a copy of this license, visit http://creativecommons.org/licenses/by/4.0/.

(C) The Author(s) 2019 